\title{
A Gas Target Internal to the LHC for the Study of pp Single-Spin Asymmetries and Heavy Ion Collisions
}

\author{
Colin Barschel, ${ }^{1}$ Paolo Lenisa, ${ }^{2}$ Alexander Nass, ${ }^{3}$ and Erhard Steffens ${ }^{4}$ \\ ${ }^{1}$ LHCb Collaboration, CERN, 1211 Geneva 23, Switzerland \\ ${ }^{2}$ University of Ferrara and INFN, 44100 Ferrara, Italy \\ ${ }^{3}$ Institut für Kernphysik, FZJ, 52425 Jülich, Germany \\ ${ }^{4}$ Physics Institute, Friedrich-Alexander University Erlangen-Nürnberg, 91058 Erlangen, Germany
}

Correspondence should be addressed to Paolo Lenisa; lenisa@fe.infn.it

Received 19 March 2015; Revised 5 June 2015; Accepted 5 July 2015

Academic Editor: Barbara Trzeciak

Copyright (C) 2015 Colin Barschel et al. This is an open access article distributed under the Creative Commons Attribution License, which permits unrestricted use, distribution, and reproduction in any medium, provided the original work is properly cited. The publication of this article was funded by $\mathrm{SCOAP}^{3}$.

We discuss the application of an open storage cell as gas target for a proposed LHC fixed-target experiment AFTER@LHC. The target provides a high areal density at minimum gas input, which may be polarized ${ }^{1} \mathrm{H},{ }^{2} \mathrm{H}$, or ${ }^{3} \mathrm{He}$ gas or heavy inert gases in a wide mass range. For the study of single-spin asymmetries in pp interaction, luminosities of nearly $10^{33} / \mathrm{cm}^{2} \mathrm{~s}$ can be produced with existing techniques.

Devoted to Professor Willy Haeberli (Wisconsin) on his 90th birthday on June 17, 2015, who presented the first ideas on a polarized storage cell target 50 years ago at the 2nd Polarization Symposium, 1965, in Karlsruhe (Germany) and who pioneered this technique and brought it to maturity

\section{Introduction}

Colliding beam facilities are indispensable tools for achieving the highest possible collision energies $\sqrt{ } \mathrm{s}$. Their range of application may be enlarged by adding a fixed-target experiment, for example, using a thin internal gas target which does not affect the luminosity life time significantly. Experiments based on such targets offer distinct features as compared with collider experiments $[1,2]$ :

(1) Accessing the large negative Feynman $\mathrm{x}_{\mathrm{F}}$ domain,

(2) achieving high luminosities with dense targets,

(3) varying the atomic number $A$ of the target in a wide range,

(4) polarizing light target atoms $\left({ }^{1} \mathrm{H},{ }^{2} \mathrm{H}=\mathrm{D},{ }^{3} \mathrm{He}\right)$ with polarization of the nuclear spin $s$.

The target proposed consists of an open storage cell, a narrow straight tube with thin walls of length $L$ located in the machine vacuum along the beam axis into which gas is injected at the center in two modes:

(i) polarized atomic beam (e.g., H) into a feed tube of low gas conductance;

(ii) unpolarized gas via capillary from a gas handling system.

The gas diffuses through the cell openings into the machine vacuum system, usually a powerful differential pumping system, and constitutes within the cell a density distribution of triangular shape with its maximum $\rho_{0}\left[\right.$ atoms $\left./ \mathrm{cm}^{3}\right]$ at the center. The areal target density $\theta$ is then $\theta=\rho_{0} \cdot L / 2$ (see Section 3). Such kind of gas targets for storage rings is reviewed in [3]. Targets for proton beams at intermediate energies have been applied at the cooler ring COSY (FZ Jülich) [4-6] and at the IUCF Cooler Ring [7]. At the $30 \mathrm{GeV}$ electron ring of the HERA collider (DESY Hamburg), the HERMES H\&D target [8] has been operated successfully during 1996-2005. In 1995, prior to the HERMES H\&D 
target, a polarized ${ }^{3} \mathrm{He}$ target [9] has been used for the measurement of the neutron spin structure function.

In the following, the design and performance of the HERMES H\&D target which may be closest in design to the proposed LHC target are reviewed in Section 2. First ideas of a storage cell target in the LHC are presented in Section 3. The paper is concluded by discussing some of the open problems which need to be subject of further studies.

\section{The HERMES Polarized Hydrogen and Deuterium Target}

An overview of the HERMES polarized $\mathrm{H}$ and $\mathrm{D}$ target [8] is presented in Figure 1. It consists of three main components:

(i) the Atomic Beam Source (ABS) for the production of an intense polarized atomic hydrogen beam, consisting of a dissociator with cooled nozzle, a system of sextupole magnets focusing the wanted hfs components $^{1}$ into the feed tube of the cell, and adiabatic rf-transitions ${ }^{2}$ for setting and switching the target polarization between states of opposite sign (Endnotes ${ }^{1,2}$ );

(ii) the target chamber with T-shaped storage cell of $400 \mathrm{~mm}$ in length and elliptical cross section with diameters horizontal/vertical of 21 and $9 \mathrm{~mm}$, respectively, and a longitudinal or vertical holding field $B$ up to $350 \mathrm{mT}$. The cell temperature could be set to values between 50 and $300 \mathrm{~K}$;

(iii) the diagnostic system for analyzing a weak sample beam drawn from the target cell, consisting of the polarimeter (BRP) measuring the substate population and the target gas analyzer (TGA) detecting the molecular fraction and thus the degree of recombination within the cell. From these parameters, the target polarization as seen by the beam is deduced.

For details of the design, operation, and analysis of the data of the diagnostic system the reader is referred to [8] and to the references therein, in particular on the various subsystems. Here a few important details are given, only. The ABS injects a flux of $\approx 6.5 \times 10^{16}$ atoms/s into the feed tube of the target cell, corresponding to a recombined $\mathrm{H}_{2}$-flow rate of about $2.5 \cdot 10^{-3} \mathrm{mbar} / \mathrm{s}$ into the machine vacuum system. The target particles $\left(\mathrm{H}, \mathrm{H}_{2}\right)$ are confined by the cell walls to the close vicinity of the beam axis, thus increasing the areal density to values of about $10^{14} / \mathrm{cm}^{2}$, two orders of magnitude higher than the density of the injected atomic beam. The Alwalls of the cell are cooled to a temperature of about $100 \mathrm{~K}$ for two reasons: (i) increasing the density $\theta$ by $\sqrt{ } 3$ and (ii) buildup of a thin layer of frozen water which suppresses wall recombination effectively. The water is produced within the dissociator from a small $\mathrm{O}_{2}$ admixture to the hydrogen gas and flows with the atomic beam.

A "strong" guide field of sufficient homogeneity is required for the target, either longitudinal for helicity measurements or transverse for transverse spin effects. The strength of about $300 \mathrm{mT}=6 \cdot B_{c}\left(B_{c}=50.7 \mathrm{mT}\right.$ is the critical field of the $\mathrm{H}$ atom) for the longitudinal field was chosen such that (i) proton and electron spins are fully decoupled and (ii) there is no depolarizing resonance due to the periodic bunch field of the beam. The target polarization ${ }^{3}$ is deduced from the data continuously recorded by the diagnostic system. The density-weighted target polarization along $z$ can be related to the sample beam data using assumptions about the relaxation mechanisms which contributes to the error.

The stability of the target is demonstrated in Figure 2, where the raw data of the transverse proton run 2002/03 are shown. For 2002, the average target polarization corrected for guide field and degree of dissociation was $\left|P_{z}\right|=0.74 \pm 0.06$ only, partly due to a less stable operation of HERA and to a weaker guide field of the normal-conducting transverse magnet, in contrast to the 1996/97 longitudinal proton run with average polarization $\left|P_{z}\right|=0.851 \pm 0.033$ (see Endnote ${ }^{3}$ ).

Polarized deuterium has been run in 1999/2000 with longitudinal field. In 2000, a very stable operation had been achieved, resulting in the following parameters: $\theta(D)=1.05$. $10^{14} / \mathrm{cm}^{2}$, with $P_{z}=+0.851 \pm 0.029$ and $-0.840 \pm 0.026$ and $P_{z z}=+0.891 \pm 0.027$ and $-1.656 \pm 0.049$ [8] (see Endnote ${ }^{3}$ ).

It should be noted that the same target has been used with unpolarized gas in a broad mass range for the study of the $A$-dependence of various effects in DIS ${ }^{4}$. The ${ }^{3} \mathrm{He}$ target [9] run in 1995 had the following characteristics: target density $\theta\left({ }^{3} \mathrm{He}\right)=3.3 \cdot 10^{14} / \mathrm{cm}^{3}$ and polarization $P_{z}=0.46 \pm 0.02$. Today, the polarization of such optically pumped targets can reach values of 0.8 , that is, $80 \%$ of the maximum [12].

\section{First Ideas on an LHC Storage Cell Target}

3.1. Density Achievable for Polarized Hydrogen. The target density $\theta$ depends on the geometry of the storage cell and the gas input. The required aperture at a beam waist may be estimated with safety margin of $1 \mathrm{~mm}$ by

$$
r=15 \sigma_{r}+1 \mathrm{~mm} \quad(r=x, y),
$$

with $\sigma_{r}$ being the $1 \sigma$ beam radius in the horizontal and vertical plane. With nominal parameters for the LHC $\mathrm{IP}^{5}$ of $\sigma^{*}=$ $16 \cdot 10^{-6} \mathrm{~m}$ and the beta function $\beta^{*}=0.55 \mathrm{~m}(*=\mathrm{min}$. values at IP), (1) results in a required aperture of $1.24 \mathrm{~mm}$, dominated by the safety margin. At $z= \pm 0.5 \mathrm{~m}$ the aperture increases slightly to $1.46 \mathrm{~mm}$. It seems unlikely that such small apertures are compatible with a stable LHC operation. Another criterion is required for first estimates on a possible cell target for the LHC.

At the VELO detector [13-15] of LHCb, the two halves can be opened during injection and tuning. In the closed position, a narrow tube of $1 \mathrm{~cm}$ in diameter and $100 \mathrm{~cm}$ in length is wide enough to guide the LHC beam through the detector. In the following we assume conservatively (see Figure 3) a size of $D_{1}=1.4 \mathrm{~cm}$ and $L_{1}=50 \mathrm{~cm}$ for a cylindrical target cell, that is, a full length of $100 \mathrm{~cm}$, which may have the option to open by being split into two halves. For the feed tube we assume $D_{2}=1.0 \mathrm{~cm}$ and $L_{2}=10 \mathrm{~cm}$, a geometry for which the HERMES ABS has been optimized. 


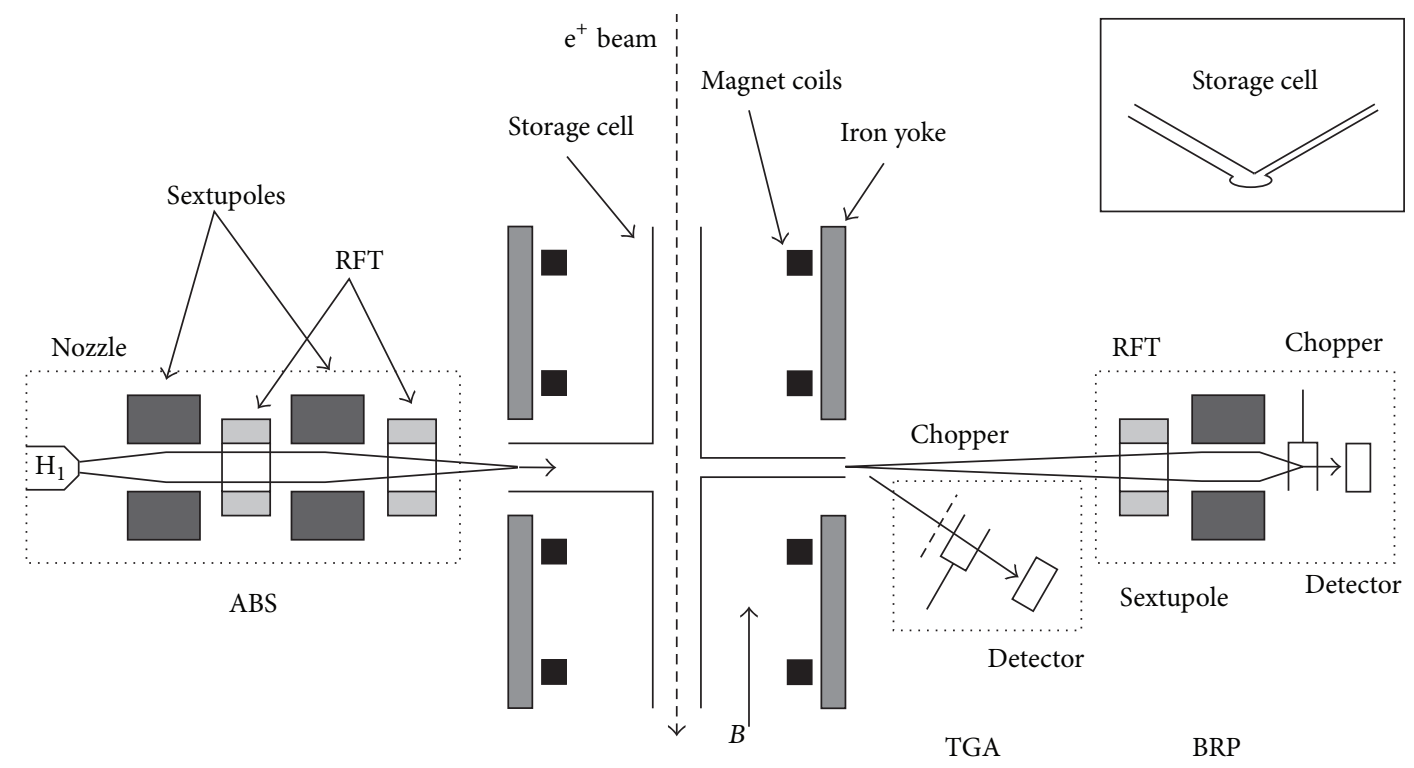

FIgURE 1: Schematic representation (top view) of the HERMES longitudinally polarized target (taken from [8]). From left to right: Atomic Beam Source (ABS), target chamber with cell and SC magnet coils, diagnostic system of target gas analyzer (TGA), and Breit-Rabi polarimeter (BRP).

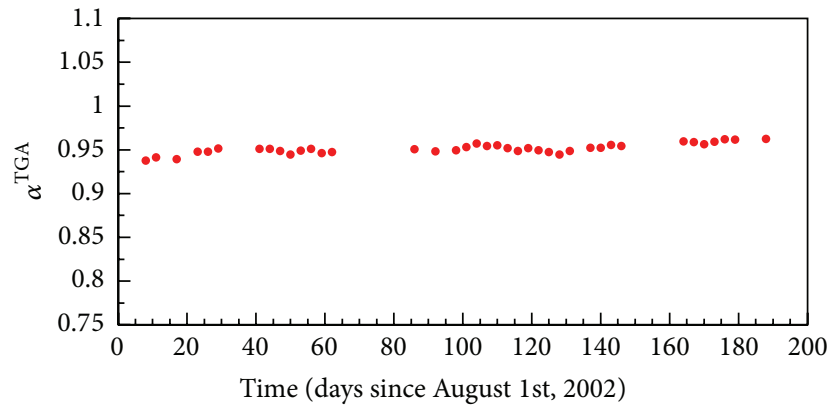

(a)

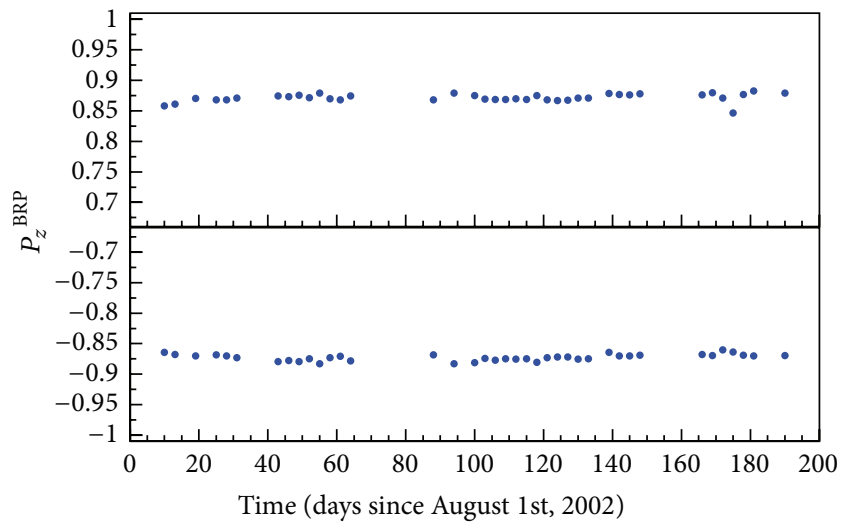

(b)

FIGURE 2: Results of the target analysis for the HERMES 2002/03 data taking period with transverse proton polarization as number of days, since August 1, 2002 (taken from [8]): (a) degree of dissociation $\alpha$ measured by the TGA ( $\alpha=1$ : no molecules); (b) positive and negative vector polarization $P_{z}$ of the sampled atomic part as measured by the BRP. During data taking the states of opposite sign are set periodically with a cycle time of the order of $1 \mathrm{~min}$.

The gas conductance of a tube in the molecular-flow regime is given by [3]

$$
C_{i}[1 / \mathrm{s}]=\frac{3.81 \sqrt{ }(T / M) D_{i}^{3}}{\left(L_{i}+1.33 D_{i}\right)},
$$

where $T$ is the temperature in $\mathrm{K}, M=1$ the molecular mass for atomic hydrogen, and $L, D$ are in $\mathrm{cm}$. The total conductance $C_{\text {tot }}$ of the cell from the center outwards is then $C_{\text {tot }}=2 C_{1}+C_{2}$ which at $T=300 \mathrm{~K}$ amounts to $C_{\text {tot }}=12.81 \mathrm{l} / \mathrm{s}$. With a flux of polarized hydrogen atoms of $I=6.5 \cdot 10^{16} \mathrm{H} / \mathrm{s}$ into the cell, the central density $\rho_{0}$ is then

$$
\rho_{0}=\frac{I}{C_{\mathrm{tot}}}=\frac{5.07 \cdot 10^{12}}{\mathrm{~cm}^{3}},
$$

resulting in an areal polarized hydrogen density of the $100 \mathrm{~cm}$ long cell of $1.4 \mathrm{~cm}$ i.d. at $T=300 \mathrm{~K}$ of

$$
\theta=\rho_{0} L_{1}=2.54 \cdot 10^{14} / \mathrm{cm}^{2} .
$$

The pp luminosity of such a target installed at LHC at nominal proton beam current of $3.6 \cdot 10^{18} \mathrm{p} / \mathrm{s}$ is then

$$
\mathscr{L}_{\mathrm{pp}}=0.92 \cdot 10^{33} / \mathrm{cm}^{2} \mathrm{~s} .
$$

For optimizing the cell wall conditions it might be necessary to run the cell at a temperature of $100 \mathrm{~K}$ instead of $300 \mathrm{~K}$, as 


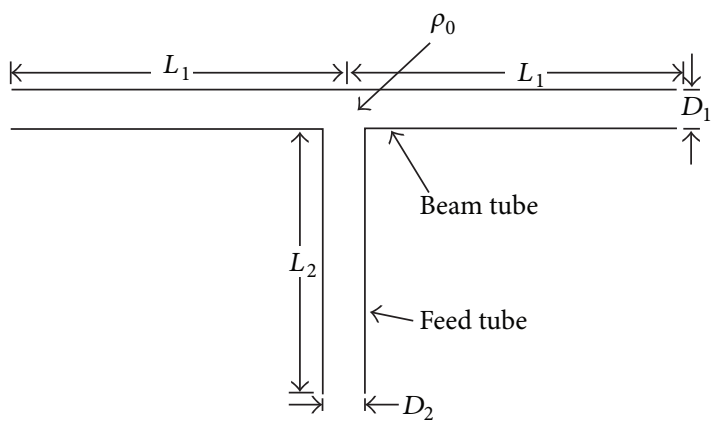

FIgURE 3: Storage cell geometry. The proton beam is traversing the beam tube of total length $2 L_{1}$; the polarized atomic beam enters "ballistically" via the feed tube into the center and diffuses outwards along the three tube elements with a density maximum $\rho_{0}$ in the center (figure taken from [3]).

for the HERMES target [8] (see Section 2). This would result in a $\sqrt{ } 3$ higher target density and a maximum luminosity of $\mathscr{L}_{\text {pp }}(100 \mathrm{~K})=1.59 \cdot 10^{33} / \mathrm{cm}^{2} \mathrm{~s}$.

If such a target is run in addition to other experiments, it is important to know its effect on the life time $\tau_{\mathrm{p}}$ of a stored LHC proton beam. Let us assume a maximum value of $\mathscr{L}_{\mathrm{pp}}=1.6 \cdot 10^{33} / \mathrm{cm}^{2} \mathrm{~s}$. The loss rate is then given by the product of $\mathscr{L}_{\mathrm{pp}}$ with the pp loss cross section at a CM energy of $162 \mathrm{GeV}$, which we estimate by $0.1 \mathrm{~b}$, resulting in a loss rate $d N / d t=N^{\bullet}=1.6 \cdot 10^{8} \mathrm{p} / \mathrm{s}$. The relative loss rate is $N^{*} / N_{\mathrm{p}}$ with the number of stored protons $N_{\mathrm{p}}=3.2 \cdot 10^{14}$, which gives a relative loss rate of $5 \cdot 10^{-7} / \mathrm{s}$, corresponding to a $1 / \mathrm{e}$ beam life time, the inverse of the relative loss rate, of $2 \cdot 10^{6} \mathrm{~s}$ or 23 days. We conclude that for the pp case additional beam losses caused by the hydrogen target gas are negligible. The target density given above is limited by the present target technology, for example, the intensity of the source feeding the cell target.

These are first estimates only, which need to be developed further if the details of a fixed-target experiment at LHC are known. The calculated target densities and the resulting luminosities are within a favorable range. Clearly, several aspects need to be investigated to make such estimates more reliable, in particular (i) the amount of target gas the LHC machine vacuum system can accept, (ii) the rôle of frozen layers like water on the inner cell surface and whether they might cause instabilities, (iii) problems of access and reliability of running a complex pumping system in the LHC tunnel, and so forth.

3.2. Density Achievable for Heavy Ion Collisions. As mentioned earlier, heavy unpolarized target gas could be injected into the cell for the study of heavy ion collisions. In principle, the gas flow from a Gas Feed System and thus the density of a cell target can be very high. On the other hand, there will be limitations due to the maximum gas flow the vacuum system at a possible LHC target station can take. Another limit might come from the rate capability of the detector system. Here, we present estimates of a density limit set by the requirement that the cell target filled with a heavy gas must not shorten the beam life time of a heavy ion beam, for example, a Pb beam, by more than $10 \%$. For the $\mathrm{LHC}$ in $\mathrm{Pb}-\mathrm{Pb}$ collider mode without gas target we assume a beam life time of $10 \mathrm{~h}$. As target gas we assume xenon with average molecular weight $M \approx 131$. As a loss cross section we start with the total hadronic cross section $\sigma_{\text {tot }}$ of $\mathrm{Pb}$ on $\mathrm{Pb}$ of $7.65 \mathrm{~b}$ [16]. By scaling with the nuclear radii, the $\mathrm{Pb}$-Xe loss cross section is estimated to be $6.6 \mathrm{~b}$. We require that the additional "target" life time ${ }^{6} \tau_{t}=10 \cdot 10 \mathrm{~h}=3.6 \cdot 10^{5} \mathrm{~s}$ is related to the the loss rate $d N / d t=N^{*}\left(N=\right.$ number of stored $\mathrm{Pb}$ ions $\left.=4 \cdot 10^{10}\right)$ by

$$
\begin{aligned}
& \frac{N^{\bullet}}{N}=\frac{1}{3.6 \cdot 10^{5} \mathrm{~s}} \\
& \text { that is } N^{\bullet}=\frac{4 \cdot 10^{10}}{3.6 \cdot 10^{5} \mathrm{~s}}=1.1 \cdot 10^{5} / \mathrm{s}=\mathscr{L}_{\mathrm{Pb}-\mathrm{Xe}} \cdot \sigma_{\text {tot }}
\end{aligned}
$$

This results in a maximum $\mathrm{Pb}$-Xe luminosity of $\mathscr{L}_{\mathrm{Pb} \text {-Xe }}=$ $1.7 \cdot 10^{28} / \mathrm{cm}^{2} \mathrm{~s}$ and a maximum density of Xenon atoms of $\theta_{\mathrm{Xe}}=3.8 \cdot 10^{13} / \mathrm{cm}^{2}$. The xenon flow rate into the target cell required to produce this density amounts to a value of 3.2 . $10^{-5} \mathrm{mbar} \mathrm{l} / \mathrm{s}$. It should be noted that the LHC $\mathrm{Pb}-\mathrm{Pb}$ design luminosity in collider mode is $1 \cdot 10^{27} / \mathrm{cm}^{2}$ s. We conclude that for the hypothetical $\mathrm{Pb}$-Xe fixed-target case a high luminosity in the order of the LHC $\mathrm{Pb}-\mathrm{Pb}$ design luminosity can be produced. The $\mathrm{Pb}$-Xe fixed-target luminosity is not limited by the storage cell technology but rather by other factors like beam life time, detector rate, and so forth.

In Table 1, the results of this study are compared with past or existing targets in storage rings, their main operational parameters, and luminosities achieved.

\section{Summary}

In this study, the application and expected performance of a storage cell target interacting with the LHC beam are investigated. Using polarized light atoms $\left({ }^{1} \mathrm{H},{ }^{2} \mathrm{D}\right.$, and possibly ${ }^{3} \mathrm{He}$ ) from external sources and a proton beam, single-spin asymmetries of various processes for longitudinal or transverse target polarization could be measured at high rate. With unpolarized atoms or molecules in a broad mass range from $\mathrm{H}_{2}$ to Xe or beyond as target gas, heavy ion collisions could be studied with flexible choice of the target mass and target density. These estimates show that a high luminosity for proton-proton and heavy ion collisions at the LHC can be achieved using the storage cell technique. The optimum density has to be determined taking a number of operational aspects into account.

It should be noted that due to the strongly forward peaked particle emission, the physics accessible by such an ultrarelativistic fixed-target experiment depends very much on the design of the detector. This is not subject of the present study.

In summary, the promising results of this study indicate that a storage cell target combined with the LHC beam may open access to additional physics topics like the gluon contribution to the proton spin, the gluon EMC effect, or 
TABLE 1: Comparison of gas targets in storage rings with a hypothetical target for the proposed AFTER@LHC initiative [1,2].The target gas ${ }^{1} \mathrm{H},{ }^{2} \mathrm{D}$, or ${ }^{3} \mathrm{He}$ is assumed to be spin polarized.

\begin{tabular}{|c|c|c|c|c|c|c|c|c|}
\hline Storage ring & Particle & $\begin{array}{c}E_{\max } \\
{[\mathrm{GeV}]}\end{array}$ & Target type & $\begin{array}{c}L \\
{[\mathrm{~m}]}\end{array}$ & $\begin{array}{l}T \\
{[\mathrm{~K}]}\end{array}$ & $\begin{array}{c}L_{\max } \\
{\left[1 / \mathrm{cm}^{2} \mathrm{~s}\right]}\end{array}$ & Remarks & Reference \\
\hline $\begin{array}{l}\text { HERA-e } \\
\text { DESY } \\
\text { (term. 2007) }\end{array}$ & $\mathrm{e}^{ \pm}$pol. & 27.6 & $\begin{array}{c}\text { Cell } \\
{ }^{1} \mathrm{H},{ }^{2} \mathrm{D},{ }^{3} \mathrm{He}\end{array}$ & 0.4 & $\begin{array}{c}100 \\
25\end{array}$ & $\begin{array}{l}2.5 \cdot 10^{31} \\
2.5 \cdot 10^{32}\end{array}$ & $\begin{array}{l}\text { HERMES exp. } \\
\text { 1995-2007 }\end{array}$ & [9] \\
\hline $\begin{array}{l}\text { RHIC-p } \\
\text { BNL }\end{array}$ & p pol. & 250 & Jet & - & - & $1.7 \cdot 10^{30}$ & $\begin{array}{l}\text { Absolute } \mathrm{p} \\
\text { polarimeter }\end{array}$ & {$[10]$} \\
\hline $\begin{array}{l}\text { COSY } \\
\text { FZ Jülich }\end{array}$ & p, d pol. & $\begin{aligned} & 3.77 \\
T= & 49.3 \mathrm{MeV}\end{aligned}$ & $\begin{array}{c}\text { Cell }{ }^{1} \mathrm{H},{ }^{2} \mathrm{D} \\
\text { Cell }{ }^{1} \mathrm{H}\end{array}$ & 0.4 & 300 & $\begin{array}{c}10^{29} \\
2.75 \cdot 10^{29}\end{array}$ & $\begin{array}{l}\text { ANKE exp. } \\
\text { PAX exp. }\end{array}$ & $\begin{array}{c}{[4,5]} \\
{[11]}\end{array}$ \\
\hline $\begin{array}{l}\text { LHC } \\
\text { CERN } \\
\text { (proposed) }\end{array}$ & $\begin{array}{l}\text { p unpol. } \\
\text { heavy ions }\end{array}$ & $\begin{array}{c}7,000 \\
2,760 \cdot A\end{array}$ & $\begin{array}{c}\text { Cell } \\
{ }^{1} \mathrm{H},{ }^{2} \mathrm{D} \\
\mathrm{Xe} \\
M \approx 131\end{array}$ & 1.0 & $\begin{array}{c}100 \\
\geq 100\end{array}$ & $\begin{array}{c}10^{33} \\
10^{27}-10^{28}\end{array}$ & $\begin{array}{l}\text { Based on techn. of } \\
\text { HERMES target }\end{array}$ & this paper \\
\hline
\end{tabular}

deconfinement studies using the $\mathrm{Pb}$ beam with targets of different mass, as discussed in $[1,2]$.

\section{Conflict of Interests}

The authors declare that there is no conflict of interests regarding the publication of this paper.

\section{Acknowledgment}

The authors thank Massimiliano Ferro-Luzzi (CERN) for valuable discussions.

\section{Endnotes}

1. Hyperfine structure components; see [3].

2. Radio-frequency transitions between hfs components; see [3].

3. The vector polarization $P_{z}$ is bound to $-1 \leq P_{z} \leq 1$ and tensor polarization $P_{z z}$ for spin $=1$ to $-2 \leq P_{z z} \leq 1$; see [3].

4. Deep inelastic scattering.

5. Interaction point, for example, collision point.

6. Note: the total target life time $\tau_{\text {tot }}$ is calculated from the individual loss rates $N_{i}^{\bullet}$ by $\tau_{\text {tot }}=\left(1 / N_{1}^{\bullet}+1 / N_{2}^{\bullet}+\cdots\right)^{-1}$.

\section{References}

[1] S. J. Brodsky, F. Fleuret, C. Hadjidakis, and J. P. Lansberg, "Physics opportunities of a fixed-target experiment using LHC beams," Physics Reports, vol. 522, no. 4, pp. 239-255, 2013.

[2] J. P. Lansberg, M. Anselmino, R. Arnaldi et al., "Spin physics and TMD studies at A Fixed-Target ExpeRiment at the LHC (AFTER@LHC)," EPJ Web of Conferences, vol. 85, Article ID 02038, 6 pages, 2015

[3] E. Steffens and W. Haeberli, "Polarized gas targets," Reports on Progress in Physics, vol. 66, no. 11, article 1887, 2003.

[4] M. Mikirtychyants, R. Engels, K. Grigoryev et al., "The polarized $\mathrm{H}$ and $\mathrm{D}$ atomic beam source for ANKE at COSY-Jülich,"
Nuclear Instruments and Methods in Physics Research A: Accelerators, Spectrometers, Detectors and Associated Equipment, vol. 721, pp. 83-98, 2013.

[5] K. Grigoryev, F. Rathmann, R. Engels et al., "Machine studies for the development of storage cells at the ANKE facility of COSY," Nuclear Instruments and Methods in Physics Research Section A, vol. 599, no. 2-3, pp. 130-139, 2009.

[6] G. Ciullo, "The H and D polarized target for spin-filtering measurements at COSY," in Proceedings of the 21st International Symposium on Spin Physics (SPIN '14), Beijing, China, October 2014, http://collaborations.fz-juelich.de/ikp/pax/documents/ talks.shtml.

[7] W. A. Dezarn, J. Doskow, J. G. Hardie et al., "Polarized internal gas target for hydrogen and deuterium at the IUCF Cooler Ring," Nuclear Instruments and Methods in Physics Research Section A, vol. 362, no. 1, pp. 36-40, 1995.

[8] A. Airapetian, N. Akopov, Z. Akopov et al., "The HERMES polarized hydrogen and deuterium gas target in the HERA electron storage ring," Nuclear Instruments and Methods in Physics Research Section A, vol. 540, no. 1, pp. 68-101, 2005.

[9] D. De Schepper, L. H. Kramer, S. F. Pate et al., "The HERMES polarized ${ }^{3} \mathrm{He}$ internal gas target," Nuclear Instruments and Methods in Physics Research A: Accelerators, Spectrometers, Detectors and Associated Equipment, vol. 419, no. 1, pp. 16-44, 1998.

[10] A. Poblaguev, "Polarimetry at the AGS," in Proceedings of the 15th International Workshop on Polarized Sources, Targets, and Polarimetry (PSTP '13), p. 053, Charlottesville, Va, USA, 2013.

[11] W. Augustyniak, L. Barion, S. Barsov et al., "Polarization of a stored beam by spin-filtering," Physics Letters B, vol. 718, no. 1, pp. 64-69, 2012.

[12] R. Milner, Private Communication, MIT, 2015.

[13] C. Barschel, Precision luminosity measurements at LHCb with beam-gas imaging [Ph.D. thesis], RWTH Aachen University, Aachen, Germany, 2014, CERN-THESIS-2013-301.

[14] M. Ferro-Luzzi, "Proposal for an absolute luminosity determination in colliding beam experiments using vertex detection of beam-gas interactions," Nuclear Instruments and Methods in Physics Research Section A, vol. 553, no. 3, pp. 388-399, 2005. 
[15] R. Aaij, C. Abellan Beteta, R. Llull et al., "Precision luminosity measurements at LHCb," Journal of Instrumentation, vol. 9, Article ID P12005, 2014.

[16] K. Oyama, "Reference cross section measurements with ALICE in $\mathrm{pp}$ and $\mathrm{Pb}-\mathrm{Pb}$ collisions at LHC," http://arxiv.org/abs/ 1305.7044 . 

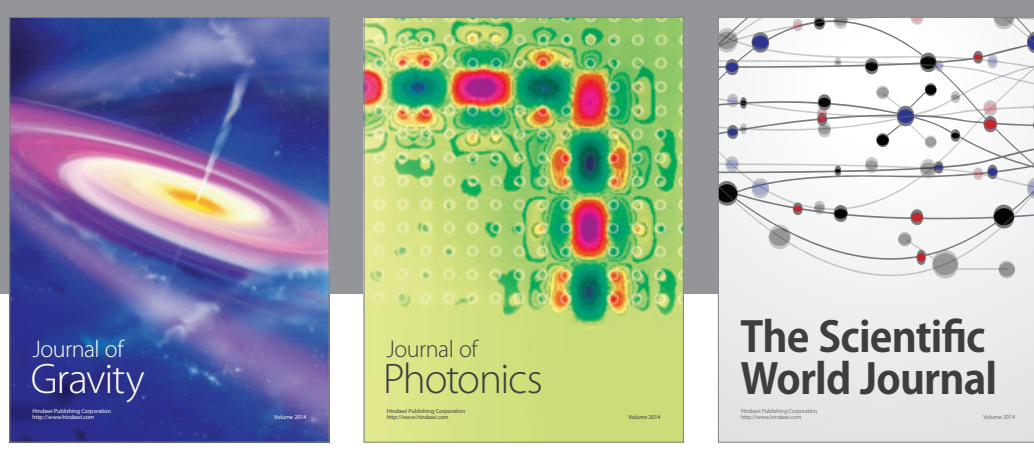

The Scientific World Journal
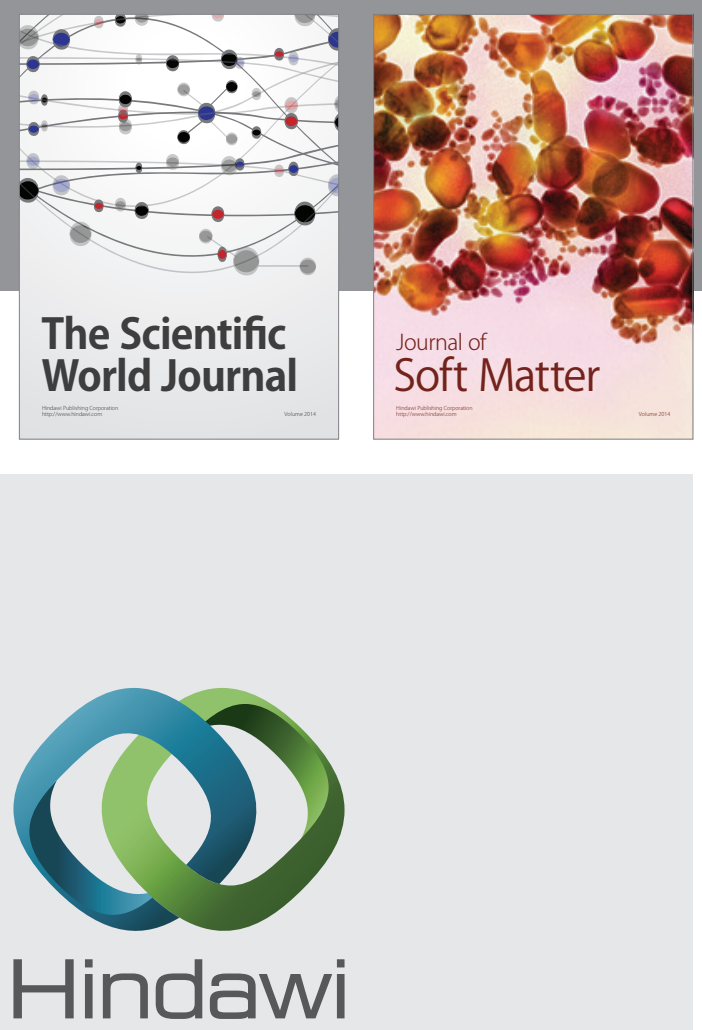

Submit your manuscripts at

http://www.hindawi.com

nternational Journal of

Statistical Mechanics
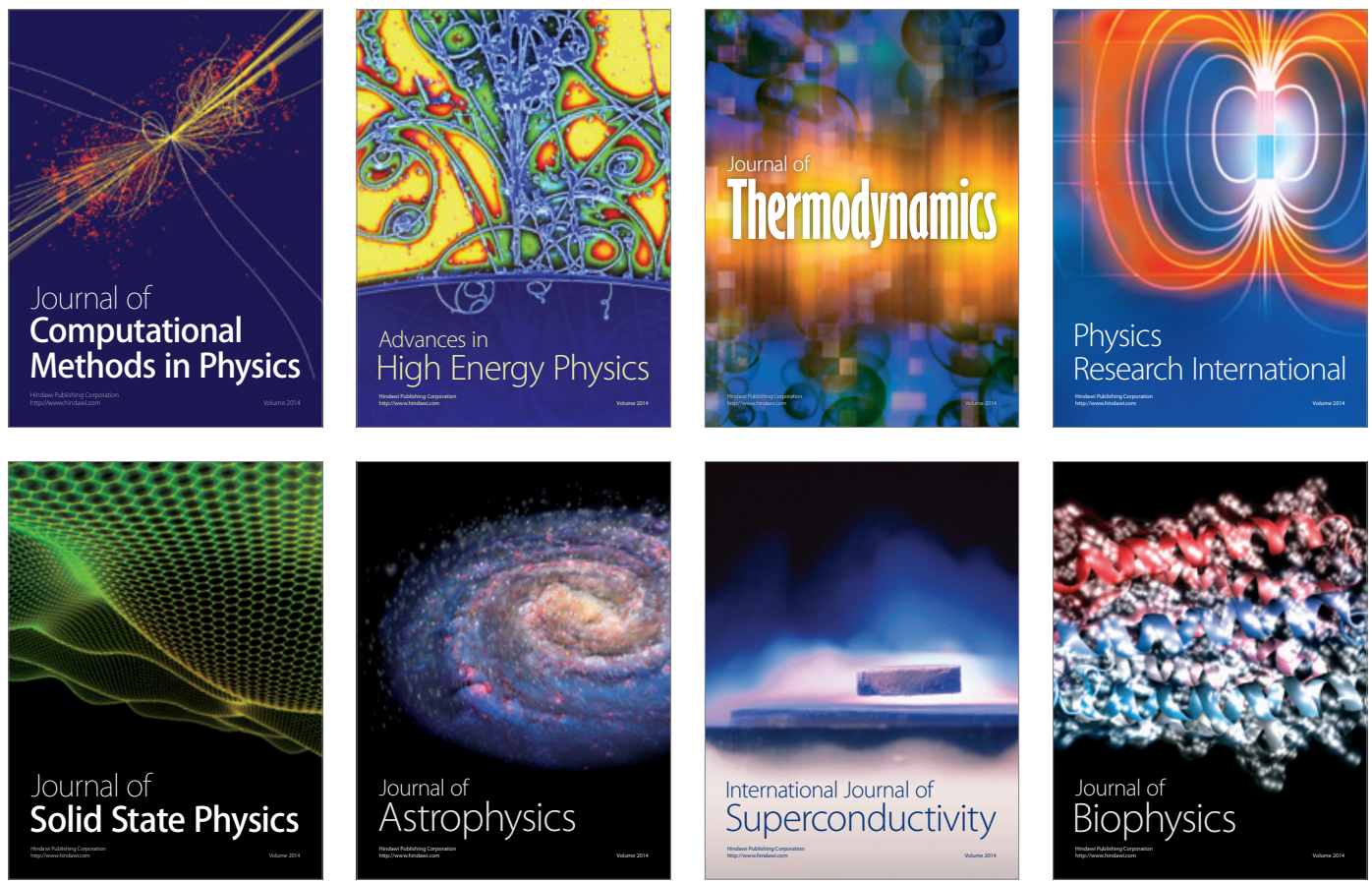
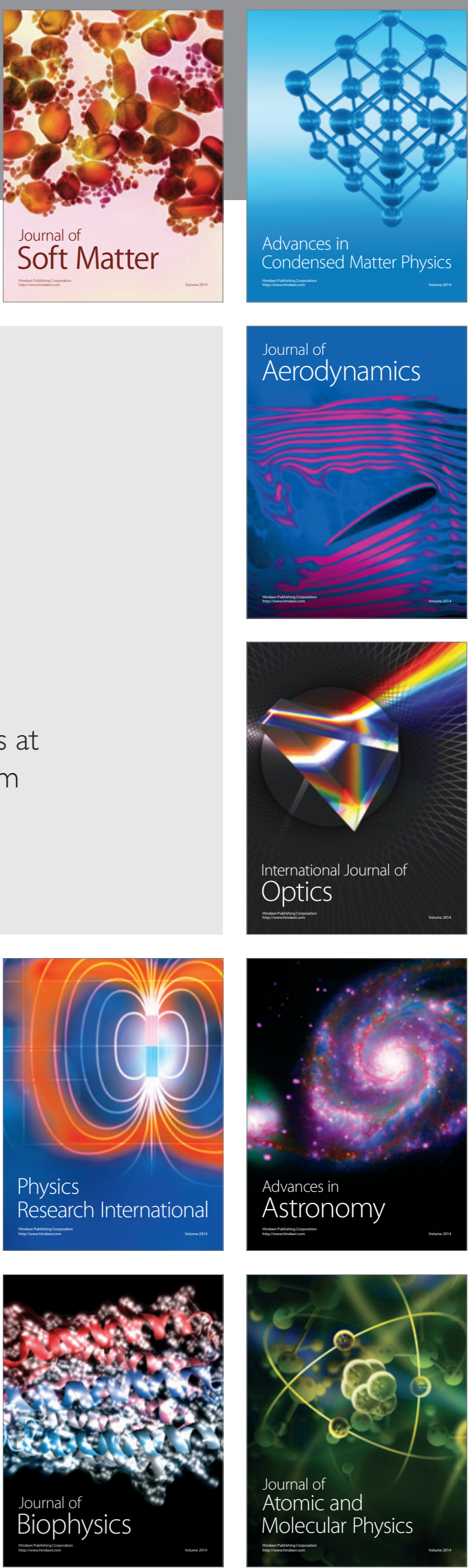